\title{
Structural Identification of Nonlinear Dynamic Systems
}

\author{
Nikolay Karabutov \\ Moscow state engineering university of information technology, radio engineering, electronics/Department of Problems \\ Control; Financial University under the Government of the Russian Federation/ Department of Mathematics, Moscow, \\ Russia \\ E-mail: kn22@yandex.ru, nik.karabutov@gmail.com
}

\begin{abstract}
The method of structural identification nonlinear dynamic systems is offered in the conditions of uncertainty. The method of construction the set containing the data about a nonlinear part of system is developed. The concept of identifiability system for a solution of a problem structural identification is introduced. The special class of structures $\mathrm{S}$ for a solution of problem identification is introduced. We will show that the system is identified, if the structure $\mathrm{S}$ is closed. The method of estimation the class of nonlinear functions on the basis of the analysis sector sets for the offered structure $S$ is described. We showed, as on $\mathrm{S}$ a preliminary conclusion about a form of nonlinear function to make. We offer algorithms of structural identification of single-valued and many-valued nonlinearities. Examples of structural identification of nonlinear systems are considered.
\end{abstract}

Index Terms - Structural Identification, Structure, Dynamic System, Nonlinearity, Secant, Algorithm, Saturation Function, Structure-Frequency Analysis.

\section{INTRODUCTION}

The problem of structural identification occupies one of the basic places in control theory. In the theory of parametric identification considerable results are received. Researches in the domain of choice of structure of model are distant from the final decision. Such condition of a problem structural identification (SI) explains complexity of mathematical statement a problem and lack of regular methods of its solution. The majority of approaches to SI are grounded on search of models from the give set or approximation of a nonlinear part system on the class of polynomials. The basis of the specified approaches is parametric identification.

Methods of an estimation structure are widely applied to the systems described by integral equations of Wiener and Wiener-Hammerstein. In [1] structure of model it is set a priori. Nonlinearity is described by polynomial function of the second order. The basic virtue of the systems described by the nonlinear equations of Wiener and Wiener-Hammerstein is grounded on model transformation to the regression to a form and application of parametric methods of identification. Such approach to identification of structure nonlinearity at the set a priori assumptions is considered in [2]. The piecewise-linear approximation is applied to nonlinearity description. The piecewise-linear method of least squares is applied to an estimation of parameters nonlinearity. Different approaches to identifi- cation of nonlinear plants on the basis of Wiener and Wiener-Hammerstein models are considered in [3, 4].

Review [5] is devoted the analysis of a condition of a problem of identification nonlinear processes in a structural dynamics. Time and frequency methods of parametric identification are considered. Methods of an estimation of type nonlinearity are analyzed. Examples of application different physical and frequency methods, and also procedures of handling results experiment for synthesis of parametric mathematical models are considered. Typical methods, applicable for studied subject domain, are considered in survey. Methods of a correlation analysis [6] and error localization in a linear model updating framework [7, 8], and also pattern recognitions [9] were applied to an estimation of type nonlinearity. The problem of an estimation of type nonlinearity solves on the class of the specified models. The problem of choice the functional form of dependence describing nonlinearity is considered. Polynomial approximation at the initial stage is offered to be applied in the presence of the a priori information. The basic problem of such approach it is choice of an order of a polynomial. Various criteria of choice of an order the polynomial, grounded on an evaluation of the significance factor [10] and coherence functions [11], are analyzed in survey. Shortages of such approach are noted. The models received by means of such approach, not always allows describing examined processes adequately. The solution of this problem gives application of Bayesian approach [12]. Limitation of the polynomial approach is noted. It does not allow describing the wide class of nonlinearities. The role of the a priori information is noted at structure choice (a nonlinearity form) noted. If the a priori information has not enough or the physical model does not reflect essence of processes it is possible to apply the concept of "a blackbox" $[13,14]$ and methods of parametric identification.

In [15] is applied nonlinear Hammerstein-type neural network model to identification of nonlinear system. The Lipschitz criterion is used for estimation an order of system. Algorithms of adjustment weights a network are offered.

A considerable quantity of publications is devoted identification of systems with a hysteresis (look, e.g. [1618]). The Bouc-Wen model (BW) is widely applied to hysteresis description. Various parametric models, approximating parameters of a hysteresis, are considered in 
[16-18]. In [16] is offered the variant Volterra/Wiener Neural Network model for an estimation of parameters a hysteresis of dynamic system the second order. The model is described by the regression equation. For identification of parameters the equation the adaptive algorithm is offered. In [17] models for approximation of a symmetric and asymmetrical hysteresis with use of piecewise-linear functions are considered. The model is described by a discrete difference equation of first order. Iterative procedure is offered for an estimation of parameters model. In [18] modification of self-adjusted differential evolutionary algorithm for an estimation of parameters the hysteresis, described BW model, is offered. Approaches to parametric identification of the hysteresis, described by various nonlinear functions, are considered in [19-21].

From review of publications follows that the overwhelming number of works is devoted problems of parametric identification of nonlinear systems subject to different various (and sometimes and full) level of the a priori information. Receive of the simplified model of nonlinearity for the purpose of its further implementation is the basis of the majority researches on nonlinearity identification. A series of researches is devoted an estimation of structure a nonlinear part system. The a priori information in these researches is used. Choice of applied methods depends on results of observations. The general approaches to a solution of a problem structural identification are not offered. One of opens problem is selection of informational set in the conditions of a priori uncertainty. This set contains the necessary data about nonlinearity. The approach offered in [22-24], is by one of perspective directions in a solution of the given problem. It allows receiving set of the data about nonlinearity in the conditions of uncertainty.

The second important problem of structural identification dynamic systems in the conditions of uncertainty is an estimation of the class nonlinear function - one-valued or multivalued. The analysis of publications displays that such researches were not fulfilled. The first results in this direction are received in [25]. In [25] method of sector sets is applied to the analysis of the special static structures reflecting behaviour of a nonlinear part a static system. The mode of construction sector sets is described and decision making procedures about the class of nonlinearity in the conditions of uncertainty of this work are offered.

In the given work development of the results received in [22-25], on nonlinear dynamic systems in the conditions of uncertainty is given. The mode of formation the informational set containing the data about a nonlinear part of system is described. We display that the given set can be generated on the basis of application the special class static models. They are received on the basis of handling to input data "input-exit". They are received on the basis of a data handling "input-output". On the basis of this set we construct the dynamic structure, reflecting in the generalized form behaviour of nonlinearity. It generalizes the structure $S_{e, k}$ offered in [23]. We describe criteria of estimation the class nonlinearity. Further we state procedures of estimation structural parameters of nonlinearity and we describe results of simulation.
We do not consider a problem of estimation an order and eigenvalues of system. They have been studied in [23].

\section{PROBLEM STATEMENT}

Consider dynamic system

$$
\begin{aligned}
& \dot{X}=F(X, U, A, t), \\
& Y=F_{Y}(X, U, A, t),
\end{aligned}
$$

where $U \in \Omega_{U} \subset \mathrm{U} \subseteq R^{m}, Y \in \mathrm{Y} \subset R^{n}$ are measured input and output of system, $X \in \mathrm{X} \subseteq R^{q}$ is a state vector, $\mathrm{Y} \subseteq \mathrm{X} \subseteq R^{n}, F: R^{q} \times R^{m} \times J \rightarrow R^{q}$ is smooth continuously differentiable on $X$ and $A$ a vector function, $A \in R^{q \times q}$ is a matrix of parameters, $t \in J \subset R$, $F_{Y}: R^{q} \times R^{m} \times J \rightarrow R^{n}$ is the function setting a mode of formation output system.

For (1) we have set of the measured data observed on a time gap $J$,

$$
\mathrm{I}_{o}=\mathrm{I}(U, Y)=\left\{U \in R^{m}, Y \in R^{n}, t \in J\right\},
$$

Problem: on the basis of the analysis and handling of set $\mathrm{I}_{o}$ to make the solution on operator structure $F$ in (1).

\section{APPROACH TO IDENTIFICATION OF CLASS NONLINEARITIES}

We will state the approach to structural identification for a special case of system (1) with the selected linear part

$$
\begin{aligned}
& \dot{X}=A X+\varphi(y) I+B u, \\
& y=C^{T} X
\end{aligned}
$$

where $u \in R, y \in R$ are input and output of system, $A \in R^{q \times q}, B \in R^{q}, I \in R^{q}, C \in R^{q}, \varphi(y)$ is some scalar nonlinear function. Consider a matrix $A$ stable.

We can make various assumptions concerning function structure $\chi=\varphi(y)$. All of them are defined by level of the a priori information. In the conditions of full a priori determinancy the methods grounded on those or other varieties of a linearization [26, 27] can be applied. In [28] the following assumption becomes concerning nonlinearity at research of an absolute stability of nonlinear systems

$$
\chi \in F_{\varphi}=\left\{\varphi(\xi) \xi \geq \xi^{2}, \xi \neq 0, \varphi(0)=0\right\},
$$

where $\xi \in R$ is an input nonlinear element of system.

Consider that is a linear combination of variables a state that is a vector. The sector condition is often used for approximation function $\chi$

$$
\begin{aligned}
\chi \in F_{\varphi}= & \left\{\gamma_{1} \xi^{2} \leq \varphi(\xi) \xi \leq \gamma_{2} \xi^{2},\right. \\
& \left.\xi \neq 0, \varphi(0)=0, \gamma_{1} \geq 0, \gamma_{2}<\infty\right\} .
\end{aligned}
$$


The analysis of works shows that in control systems static nonlinearities are often applied. Therefore we will apply the models described by the static (algebraic) equations to a function estimation $\varphi(\cdot)$. We state that in the conditions of a priori uncertainty we have the data which do not allow solving a problem structural identification $\varphi(\cdot)$. Therefore, we for identification of this class models should obtain a corresponding subset of the measured data or their converted analogue.

We suppose that the set $I_{o}$ for system (3) have the form

$$
\mathrm{I}_{o}=\left\{u(t), y(t) \quad t \in J=\left[t_{0}, t_{k}\right]\right\} .
$$

Problem: on the basis of a data handling (6) estimate the class to which belong nonlinear function $\varphi(y)$ in (3) and its structural parameters.

For a solution of a problem we use methodology of informational synthesis [22, 23]. It realizes following steps.

1. Formation of the set $\mathrm{I}_{N, g}$ containing the data about a nonlinear part of system (3).

2. Construction of the structures $S$ reflecting in the generalized form of property a nonlinear part system on the basis of the analysis $\mathrm{I}_{N, g}$.

3. Development of algorithm decision making about the class of nonlinearity $F_{\varphi}$ on the basis of the analysis $S$.

4. Development of procedure of estimation parameters of nonlinearity on the class $F_{\varphi}$.

So, pass to the first step of a solution of a problem structural identification.

\section{SET FORMATION $\mathrm{I}_{N, g}$}

Apply to $y(t)$ operation of differentiation and designate the received variable as $x_{1}$. The account $x_{1}$ gives expansion of informational set $\mathrm{I}_{o}: \mathrm{I}_{e n t}=\left\{\mathrm{I}_{o}, x_{1}\right\}$.

Remark 1. If variables $u, y$ are measured with an error at first apply filtering or smoothing procedure.

Select the subset of the data $\mathrm{I}_{g} \subset \mathrm{I}_{e n t}$ corresponding to a particular solution of system (3) (steady state), for realization of the first step procedure. We form this set deleting the data $\mathrm{I}_{t r}$ containing the information on transient process in system, that is $\mathrm{I}_{g}=\mathrm{I}_{e n t} \backslash \mathrm{I}_{t r}$.

Apply mathematical model

$$
\hat{x}_{1}^{l}(t)=H^{T}\left[\begin{array}{lll}
1 & u(t) & y(t)
\end{array}\right]^{T}
$$

to selection of the linear component $x_{1}$ defined on the interval $J_{g}=J \backslash J_{t r}$. Here $H \in R^{3}$ is a vector of parameters model.

We define a vector $H$ from a condition of minimization squared criterion $Q(e)=0.5 e^{2}$

$$
\left.\min _{H} Q(e)\right|_{e=\hat{x}_{1}^{l}-x_{1}} \rightarrow H_{o p t} .
$$

On the basis of the model (7) define the forecast for a variable $x_{1}(t) \quad \forall t \in \mathrm{I}_{g}$ and generate an error $e(t)=\hat{x}_{1}^{l}(t)-x_{1}(t) . e(t)$ depends on nonlinearity $\varphi(y)$ in system (3). As a result we receive set

$$
\mathrm{I}_{N, g}=\left\{y(t), e(t) t \in J_{g}\right\}
$$

which we will apply at the second stage of informational synthesis. Further we will use a designation $y(t)$, supposing that $y(t) \in \mathrm{I}_{N, g}$.

\section{STRUCTURES $S_{e y}, S_{e k}$}

We will consider a phase portrait of system (3) in space $\mathscr{P}_{y e}=(y, e)$ for the analysis of properties nonlinear systems. To phase portrait there corresponds the structure $S_{e y}$ described by the function $\Gamma_{e y}:\{y\} \rightarrow\{e\} \quad \forall t \in J_{g}$. $S_{e y}$ can have the closed form. It $S_{e y}$ differs from structures $S_{e k}$ which are applied to the analysis of static systems [23, 24].

We will use to decision making also $S_{e k}$-structure which is described by function $\Gamma_{e k}:\left\{k_{s}(t)\right\} \rightarrow\{e(t)\}$, where $k_{s}(t) \in R$ is a coefficient of structural properties $[23,24]$ systems in space $\mathcal{P}_{y e}$

$$
k_{s}(t)=\frac{e(t)}{y(t)} .
$$

Results of simulation will show that many approaches offered for an identification of structure static systems [24], are applicable and for dynamic systems. We can a solution about the class of nonlinear functions (onevalued or multivalued) in a system (3) to accept on the basis of the application results work [24]. Further, we describe the corresponding approach.

As we analyze an identification problem at first we will consider the problem on identifiability of system (2). We give a solution of this problem on the basis of the analysis properties the offered structures.

\section{About PROPERTIES $I_{N, g}$}

Consider properties of set $\mathrm{I}_{N, g}$ at which the solution of a problem structural identification function $\varphi(y)$ is possible. At first, the initial set $\mathrm{I}_{o}$ should allow to solve a problem of parametric model identification (7). It means that the input $u(t)$ should be nondegenerate on the interval $J$. Secondly, the input $u(t)$ should give informative structure $S_{e y}\left(\mathrm{I}_{N, g}\right)$ (or $\left.S_{e k}\right)$. It means that we the make a decision about nonlinear properties of a system (3) on the 
basis of the analysis can $S_{e y}$. Such input we will name representative.

Let the structure $S_{e y}$ is closed. Let $h\left(S_{e y}\right)$ is a distance between two points the structure opposite sides $S_{e y}$.

Statement 1. Let: 1) the linear part of system (3) is stable to, and nonlinearity $\varphi(\cdot)$ will satisfy to a condition (5);2) the input $u(t)$ is restricted piecewise continuous and extreme nondegenerate; 3) exist such $\delta_{S}>0$ that $h\left(S_{e y}\right) \geq \delta_{S}$. Then the structure $S_{e y}$ is identified on set $\mathrm{I}_{N, g}$.

Proof. Consider an input $u(t)$, satisfying to a condition 1) $u(t)$ corresponds a Fourier series containing of a sinusoid frequency $\omega_{i}$ of a frequency spectrum $u(t)$. The output $y(t) \in \mathrm{I}_{N, g}$ also contains components of this spectrum and has phase shift in relation to $u(t) . x_{1}$ is a result of differentiation $y(t)$. Hence $x_{1}$ contains components with the specified frequency spectrum. Therefore the structure $S_{e y}$ on a phase plane $\left(y, x_{1}\right)$ has the closed character. $S_{e y}$ is closed. Therefore we can define distance $h\left(S_{e y}\right)$ between opposite points of structure. $y(t), x_{1}(t)$ are extreme nondegenerate functions of a time according to a condition 1). Therefore $h\left(S_{e y}\right)>\delta_{S}$ for almost all $t \in J_{N, g}$.

The structure $S_{e y}$ having specified properties, we name $h$-identified. Further we suppose that $S_{e y}$ has the specified property.

Consider concept features $h$-identifiability.

1. $h$-identifiability is applied to a solution nonparametric, and to structural identification.

2. The demand of parametric identifiability is the fundamentals $h$-identifiability.

3. $h$-identifiability specifies more rigid demands to a system input.

The feature 3 means that "the bad" input can fulfil to an extreme nondegenerate condition. But it can give socalled, insignificant $S_{e y}$-structure which will have as before property $h$-identifiability. Property of insignificance in the conditions of uncertainty can bring to an identification of nonlinearity, atypical for researched system. This problem demands more detailed study.

Now we will pass to an estimation of the class of nonlinearity $\varphi(y)$. It is one of the main problems of structural identification.

\section{ESTIMATION OF CLASS NONLINEARITY}

We will consider classes of single-valued $F_{o v}$ and many-valued $F_{m v}$ nonlinearities. These classes contain the big set of nonlinear functions. Fulfil a fragmentation of structure $S_{e y}$ on the basis of use a subset $\mathrm{I}_{\varphi} \subseteq \mathrm{I}_{N, g}$ for an estimation of the class nonlinearities. $\mathrm{I}_{\varphi}$ adequately reflects a variation of a function $\chi=\varphi(y)$ in structural space $\mathscr{P}_{y e}$.

The problem of finding a subset $\mathrm{I}_{\varphi} \subseteq \mathrm{I}_{N, g}$ is nontrivial and depends on the available a priori information [25]. It is reduced to selection of such interval a modification a variable $y \in \mathrm{I}_{\varphi} \subseteq \mathrm{I}_{N, g}$ in the conditions of uncertainty on which we see features of function $\chi$. We will select $\mathrm{I}_{\varphi} \subseteq \mathrm{I}_{N, g}$ on the basis of the analysis of a change of structure $S_{e y} \cdot \mathrm{I}_{\varphi}$ correspond fragments $F R_{\varphi} \subset S_{e y}$ on which we see change features $S_{e y}$.

On the basis of the analysis fragments $F R_{\varphi} \subset S_{e y}$ select a subset of intervals $\mathrm{I}_{y}^{j}(j=\overline{1, s}) . \mathrm{I}_{y}^{j}$ are basis for the analysis of features $\varphi(y)$. Intervals $\mathrm{I}_{y}^{j}$ not linked with features of nonlinear function can contain fragments. We fathom under features of function loss of continuity, flex points and extremes on some interval $\mathrm{I}_{y}^{j}$. All these features are an indication of nonlinearity examined function. In this sense we will use concept of self-descriptiveness of set $\mathrm{I}_{y}^{j}[23,24]$.

Statement 2. Let on some interval $\mathrm{I}_{y}^{j}$ functions

$$
\left(\mu_{e}^{j}(t)=\frac{d e(t)}{d y}\right) \vee\left(\mu_{k_{s}}^{j}(t)=\frac{d k_{s}(t)}{d y}\right) \quad \forall j=\overline{1, s}
$$

are not continuous or in their behaviour there are features. Then the interval $\mathrm{I}_{y}^{j}$ can be considered as informative.

This statement does not envelop some classes of nonlinear functions. Such approach is justified in the conditions of a priori uncertainty. The specified property of self-descriptiveness depends from $h$-identifiabilities of structure $S_{e y}$.

We on the basis of the analysis a change parameters (8) on each of $J_{g}^{i}$ eliminate spurious subsets $\mathrm{I}_{y}^{j}$ from consideration. Remained $\mathrm{I}_{y}^{j}$ include in composition $\mathrm{I}_{\varphi}$

$$
\mathrm{I}_{\varphi}=\bigcup_{i}^{v} \mathrm{I}_{\varphi}^{i}\left(\mathrm{I}_{y}^{i}\right), \quad v \leq s .
$$

So, we have received informational set $\mathrm{I}_{\varphi} \subseteq \mathrm{I}_{N, g}$. $\mathrm{I}_{\varphi}$ allows passing to an estimation of the class nonlinearity $\varphi(y)$.

Consider a fragment $F R_{\varphi}^{i} \subset S_{e y}$ defined on $\mathrm{I}_{\varphi}^{i}$ for $\exists i \geq 1$. Construct for $\mathcal{F R}_{\varphi}^{i}$ sector set [25]. Apply a leastsquares method and define a secant $\bar{\gamma}_{i}$ for $\mathcal{F R}_{\varphi}^{i}$ on $\mathrm{I}_{\varphi}^{i}$

$$
\bar{\gamma}_{i}=\bar{\gamma}(y(t))=a_{i} y(t)+b_{i},
$$

Find mean value $\bar{y}_{i}$ for $y(t)$ on $\mathrm{I}_{y}^{i} \subset \mathrm{I}_{\varphi}$. Let $\bar{y}_{i}$ is a 
centre $\mathcal{F R}_{\varphi}^{i}$ on $\mathrm{I}_{y}^{i} \subset \mathrm{I}_{\varphi}$. Draw a perpendicular from a point $\bar{y}_{i}$ to intersection with $\bar{\gamma}_{i}$ on a plane $(y, e)$. Set magnitude $c_{i}>0$ and in a point $\alpha=\left(\bar{y}_{i}, e\left(\bar{y}_{i}\right)\right)$ draw straight lines

$$
\bar{\gamma}_{i,-}=a_{i,-} y(t)+b_{i}, \bar{\gamma}_{i,+}=a_{i,+} y(t)+b_{i},
$$

where $a_{i,+(-)}=a_{i} \pm c_{i}$.

Name $\operatorname{Sec}_{a_{i}}\left(F R_{\varphi}^{i}\right)=\left(\bar{\gamma}_{i,-}, \bar{\gamma}_{i,+}\right)$ sector set for $F R_{\varphi}^{i}$. Let $\operatorname{Sec}\left(F R_{\varphi}^{i}\right)=\operatorname{Sec}_{\alpha, l}\left(F R_{\varphi}^{i}\right) \cup \operatorname{Sec}_{\alpha, r}\left(F R_{\varphi}^{i}\right)$,

where $\operatorname{Sec}_{\alpha, l}\left(F R_{\varphi}^{i}\right), \operatorname{Sec}_{\alpha, r}\left(F R_{\varphi}^{i}\right)$ are the subsets $\operatorname{Sec}\left(F R_{\varphi}^{i}\right)$ arranged at the left and to the right of a point $\alpha$.

We secants

$$
\bar{\gamma}_{i, l}=a_{i, l} y(t)+b_{i, l}, \bar{\gamma}_{i, r}=a_{i, r} y(t)+b_{i, r}
$$

for each of parts $F R_{\varphi, l(r)}^{i}$ a fragment $F R_{\varphi}^{i}$, belonged $\operatorname{Sec}_{\alpha, i}\left(F R_{\varphi}^{i}\right)$ and $\operatorname{Sec}_{\alpha, l(r)}\left(F R_{\varphi}^{i}\right)$, will define. Further we will apply modification of statement from [25].

Let exist such $\delta_{i}>0$ that

$$
\left|a_{i, l}-a_{i}\right| \leq \delta_{i}, \quad\left|a_{i, r}-a_{i}\right| \leq \delta_{i} .
$$

Theorem 1. Let for system (3) in space $\mathscr{P}_{y e}=(y, e):$ i) structures $F R_{\varphi, r}^{i}, F R_{\varphi, l}^{i}$ described by maps

$$
\Gamma_{e y, l(r)}:\{y\}_{i, l(r)} \rightarrow\{e\}_{i, l(r)},
$$

where $\{y\}_{i, l(r)} \subset \mathrm{I}_{\varphi}^{i},\{e\}_{i, l(r)} \subset \mathrm{I}_{\varphi}^{i}$, and secants (10) corresponding to them are received; ii) for $F R_{\varphi}^{i}$ the secant (9) is received. Then: 1) function $\varphi(y) \in F_{o v}$ if (11) is fulfilled; 2) function $\varphi(y) \in F_{m v}$ if (11) is not fulfilled.

The proof of the theorem 1 is analogous to the proof of statement from [25] and is grounded on homothety application.

From the theorem 1 follows that if conditions (11) are fulfilled, for $\varphi(y)$ true a Holder-Lipschitz condition and to sectors $\operatorname{Sec}_{\alpha, l}\left(F R_{\varphi}^{i}\right), \operatorname{Sec}_{\alpha, r}\left(F R_{\varphi}^{i}\right)$ operation of a homothety [29] is applicable.

Other approach to an estimation of the class nonlinearity $F_{\varphi}$ is founded on the construction of a sector for $\varphi(y)$. We will apply the approach offered in [25] to its realization. In this case pass in space $\mathscr{P}_{k e}=\left(k_{s}, e\right)$.

Now we will consider methods of identification structure (form) of the function $\varphi(y)$ belonging to the set class $F_{\varphi}$.

VIII. ESTIMATION STRUCTURE $\varphi(y)$
The problem of structural identification of nonlinear systems is difficult and complicated. We cannot offer the common approaches. Each class of nonlinearity $F_{\varphi}$ has the features. They affect behaviour of trajectories of system, and, therefore, influence properties of structure $S_{e y}$. Detection of these features gives the detailed analysis $S_{e y}$ in the conditions of uncertainty. Therefore further, we will consider concrete examples of systems with $\varphi(y) \in F_{\varphi}$ to state identification methodology. The result of such approach is a development of algorithm identification structure $\varphi(y)$ for the concrete class $F_{\varphi}$.

\section{A. Class $F_{o v}$}

In space $\mathscr{P}_{y e}$ consider structure $S_{e y}$ and a fragment $F R_{\varphi}^{i} \subset S_{e y}$ defined on $I_{y}^{i} \subseteq \mathrm{I}_{\varphi}^{i}$. We suppose that on $I_{y}^{i} \subseteq \mathrm{I}_{\varphi}^{i}$ conditions (11) are satisfied. Therefore nonlinearity is described by a monotone function. We suppose that the fragment $F R_{\varphi}^{i} \subset S_{e y}$ is $h$-identified.

We will explain the approach to SI on an example of system the second order (3) with following parameters

$$
\begin{aligned}
& A=\left[\begin{array}{cc}
0 & 1 \\
-3 & -4
\end{array}\right], x(0)=2, x^{\prime}(0)=1, \\
& u(t)=5+2 \sin (0.1 \pi t), \varphi(x)=x^{0.4}, y=x .
\end{aligned}
$$

The system was integrated on the interval $[0 ; 30] \mathrm{s}$ with a step 0,2s. We have generated sets $\mathrm{I}_{o}, \mathrm{I}_{N}, \mathrm{I}_{N, g}$. We showed on Fig. 1 phase portrait of system and structure $S_{e y}$ on the interval $J_{g}=[5,4 ; 27,2] \mathrm{s}$. On $S_{e y}$ we have selected two fragments $F R_{\varphi}^{i} \in F_{o v}, i=1,2$. The Fig. 1 confirms identifiability of structure $S_{e y}$.

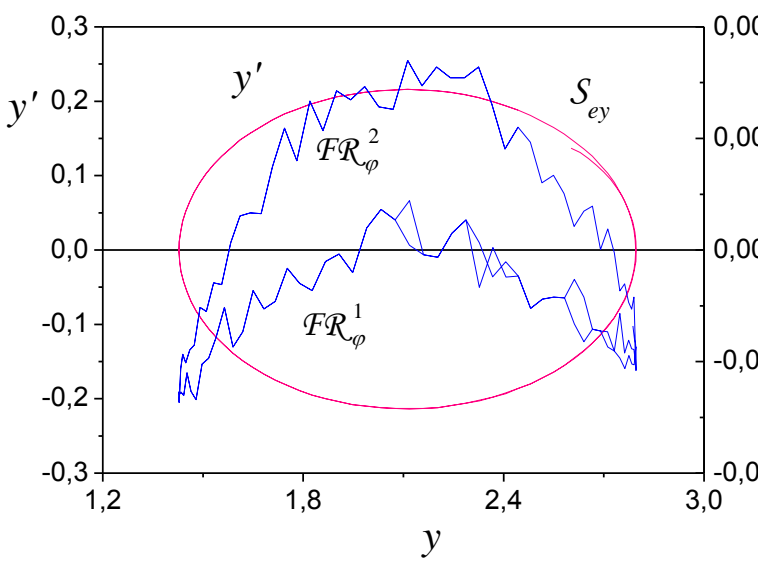

Fig. 1. Phase portrait of system and $S_{e y}$-structure

From Fig. 1 nonlinearity of system follows. We will pass from $S_{e y}$ to structure $S_{e k}$ for simplification of procedure decision making about a form $\varphi(y)$.

On Fig. 2 we will show that $\varphi(x) \in F_{o v}$. We define sec 
tor $\operatorname{Sec}_{k}(e)$ for $S_{e k}$ and secants for $F R_{\varphi, k}^{1}, F R_{\varphi, k}^{2}$. Curves 1, 2 on Fig. 2 correspond $F R_{\varphi, k}^{1}, F R_{\varphi, k}^{2}$. We will not show on Fig. 2 structure $S_{e k}$, as $S_{e k}=F R_{\varphi, k}^{1} \cup F R_{\varphi, k}^{2}$. This note is fair also sectors $\operatorname{Sec}\left(F R_{\varphi, k}^{1}\right), \operatorname{Sec}\left(F R_{\varphi, k}^{2}\right)$ as they practically coincide with boundaries $\operatorname{Sec}_{k}(e)$.

Secants for $S_{e k}$ and $F R_{\varphi, k}^{1}, F R_{\varphi, k}^{2}$

$$
\bar{\gamma}_{k}=1,83 k_{s}, \bar{\gamma}_{1, k}=1,849 k_{s}, \bar{\gamma}_{2, k}=1,87 k_{s} .
$$

Coefficients of determinations for secants exceed 0.925 . Application of the theorem 1 with $\delta_{1}=\delta_{2}=0.05$ gives $\varphi(x) \in \mathcal{F}_{o v}$.

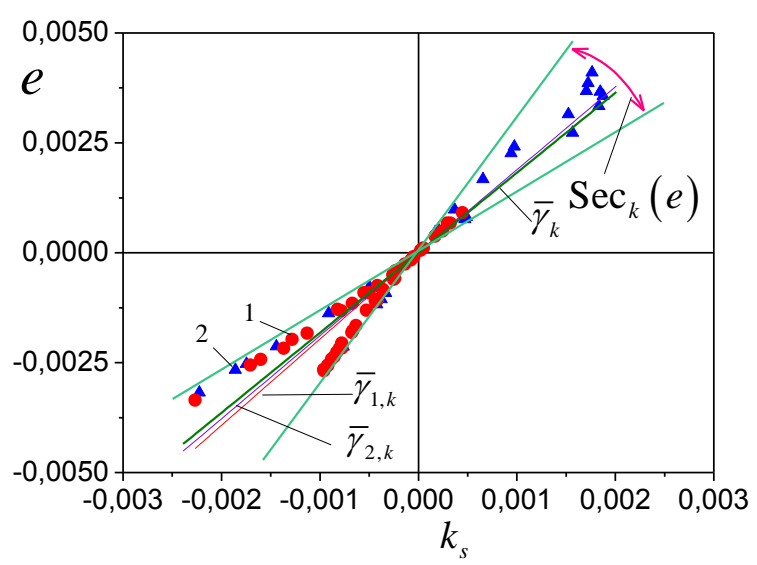

Fig. 2. Sector for $\varphi(x)$

Now we will consider a problem of identification a form function $\varphi(x)$. Select on $S_{e k}$ the fragment $F R_{\varphi, k}^{2} \subset S_{e k} \quad$ defined on a subinterval $J_{g, k}=[16,4 ; 26,2] \mathrm{s}$. It is showed on Fig. 3. $F R_{\varphi, k}^{2} \subset S_{e k}$ corresponds a fragment $\mathrm{FR}_{\varphi}^{2} \subset S_{e y}$.

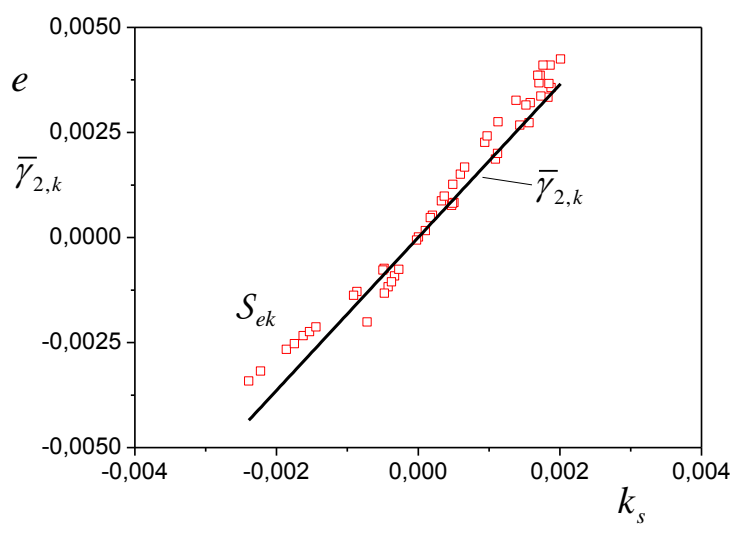

Fig. 3. $S_{e k}$-structure

On Fig. 3 we showed a secant $\bar{\gamma}_{2, k}(t)=a_{2, k} k_{s}(t)$ for $F R_{\varphi, k}^{2}$. The coefficient of determination for $\bar{\gamma}_{2, k}$ is $r_{e, \bar{\gamma}_{2, k}}^{2}=0,956$. So, as a first approximation we have a linear estimator for $\varphi(x)$. Verify the obtained result.

We will apply offered in [30] to statics systems the approach for decision making about a form $\varphi(y)$. Approach implementation is grounded on following operations. At first we introduce criterion of decision making. Further we select the structure function $\varphi(y)$, satisfy to this criterion. We apply for this purpose the theorem 3.7 [30].

Construct for $S_{e y}$ a secant described by a polynomial of order $p$,

$$
\bar{\gamma}_{2, k, p}\left(e^{v}, k_{s}\right)=\bar{\gamma}_{2, k, p}=a_{0,2, p}+\sum_{j=1}^{p} a_{1, j, p} k_{s}^{j} .
$$

Order of a polynomial (12) define from a condition

$$
\max _{j} r_{e \bar{\gamma}_{2, k, p}}^{2}(j) \rightarrow p^{*},
$$

where $r_{e \bar{\gamma}_{2, k, p}}^{2}$ is a coefficient of determination between $e$ and $k_{s}$. Results of simulation give value $p=3$ with $r_{e \bar{\gamma}_{2, k, p}}^{2}=0.982$.

Consider the class of elementary functions. At first apply a power function $y^{\pi}$ in the capacity of the candidate on $\varphi(y)$. Construct secant $S_{e k}$ as function from $y^{\pi}$

$$
\bar{\gamma}_{2, k, \pi}(t)=a_{2, k, \pi} k_{s}(t, \pi),
$$

where $k_{s}(t, \pi)=e(t) / y^{\pi}(t)$.

At $\pi=0.6$ we obtain $r_{e \bar{\gamma}_{2, k .0 .6}}^{2}=0.987$, and at $\pi=0.4$ $r_{e \bar{\gamma}_{2, k, 0.4}}^{2}=0.994$. Apply the theorem 3.7 [30] and choose a power function with $\pi=0.4$.

Remark 2. The described approach to secant choice $\bar{\gamma}_{2, k, \pi}$ in [23] is named by a straightening method. The graphic-analytical approach analogous to a method of straightening was applied in [31].

Coefficients of determinations secants with nonlinear functions $\sin (y), \ln (y), e^{y}$ are less magnitude $r_{e \bar{\gamma}_{2, k, p}}^{2}$. Analogous results are true and for a fragment $F R_{\varphi, k}^{1} \subset S_{e k}$.

Now we will generalize the obtained results and the approach offered in [30]. We offer the following algorithm of choice structure function $\varphi(y)$ on the class $F_{o v}$.

1. Define structure $S_{e y}$ and estimate identifiability of system on $S_{e y}$.

2. Construct sector $\operatorname{Sec}\left(F R_{\varphi}^{i}\right)$ for $F R_{\varphi}^{i} \subseteq S_{e y}$. If it is necessary, fulfil this step for $S_{e k}$.

3. Make a solution on the class $F_{o v}$. If $\varphi(y) \in F_{o v}$, go to a step 4.

4. Go into structural space $\mathscr{P}_{k e}=\left(k_{s}, e\right)$ and construct structure $S_{e k}$. 
5. On $S_{e k}$ select a fragment $F R_{\varphi, k}^{i} \subset S_{e k} i \geq 1$.

6. Define for $\mathcal{F R}_{\varphi, k}^{i}$ a secant $\bar{\gamma}_{i, k}$ and a coefficient of determination $r_{e, \bar{\gamma}_{i, k}}^{2}$.

7. Define a secant $\bar{\gamma}_{2, k, p}(12)$, and parameter $p$ will select from a condition (13).

8 . Set the class of the elementary single-valued nonlinearities. Use a method of straightening and choose structure of function $\varphi(y)$.

Sometimes in space $\mathscr{P}_{k e}$ it is not possible to make a solution on a form of function $\varphi(y)$. It is true for power and sinusoidal functions. In this case be switched in space $\mathscr{P}_{e y}$ and make a solution on the basis of the structure analysis $S_{e y}$.

\section{B. Class $F_{m v}$}

Consider now the class of many-valued functions. We use for a solution of a problem structure $S_{e y}, S_{e k}$. The structure $S_{e k}$ unlike $S_{e y}$ allows to find parameters function $\varphi(y)$.

We will explain the approach to structure choice $\varphi(y)$ on an example of system the second order considered in section 8.1. Input is $u(t)=2 \sin (0.1 \pi t) \cdot \varphi(y)$ is described by saturation function

$$
\varphi(y)=\operatorname{sat}(y)=\left\{\begin{array}{l}
\bar{v}, \quad y>1, \\
\kappa y, y \leq 1, \quad \bar{v}=2, \kappa=2, \underline{v}=-1 . \\
\underline{v}, y<-0.5,
\end{array}\right.
$$

Corresponding structures for a steady state are showed on Fig. 4.

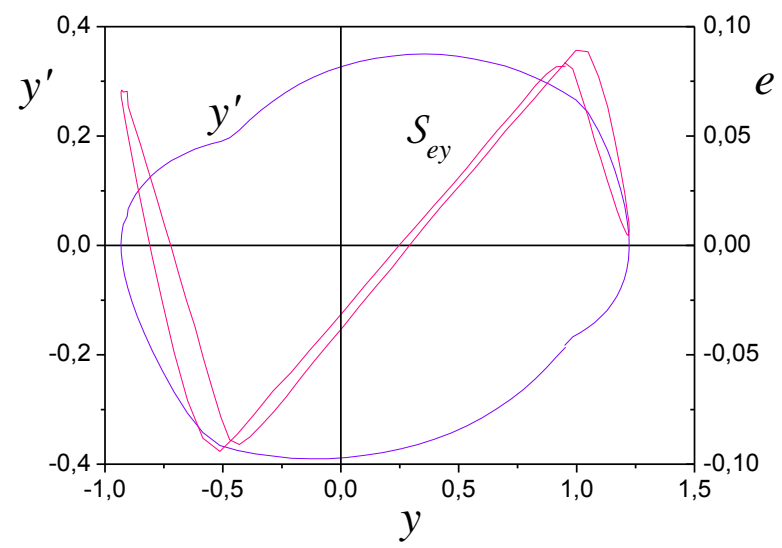

Fig. 4. Structures of system the second order with saturation

From Fig. 4 we see that in points $y=-0.5, y=1$ the system changes the properties. The analysis of behaviour structure will show that in neighbourhood of the specified points is had a sharp change of properties. On the interval $(-0.5 ; 1)$ we see linear growth $e(t)$. From $S_{e k}$ follows, that we observe sharp growth (slope) $y^{\prime}(t)$ out of the specified interval. Such behaviour of a system we see on the ends of variations intervals of a variable $y(t)$. Compare such behaviour of a system to presence at it a condition of cutting off (saturation).

We do not state a method of estimation the class $F_{\varphi}$ for considered function. It is described in section 7.A.

So, the visual analysis even in the conditions of uncertainty allows making preliminary assumptions about a nonlinearity form $\varphi(y)$.

Pass to an estimation stage of parameters. We will divide the upper part of the structure $S_{e y}$ defined on a time gap $[17.2 ; 27.4] \mathrm{s}$, on fragments $F R_{\varphi}^{i}, i=1,2,3$. They are showed in Fig. 5. Fragments $F R_{\varphi, k}^{i}$ correspond $F R_{\varphi}^{i}$ on $S_{e k}$. Further, we will apply the approach described in section 7.A. Consider a fragment $F R_{\varphi, k}^{2} \subset S_{e k}$ for definition of an angle of inclination function sat $(y)$. Apply secant method. Obtain for an angle of inclination $\operatorname{sat}(y)$ an estimation 2.03. The linear part $\operatorname{sat}(y)$ is defined on the interval $[-0.5 ; 1]$. Therefore for saturation areas $\operatorname{sat}(y)$ obtain estimations $\hat{\bar{v}}=2, \underline{\hat{v}}=-1$.

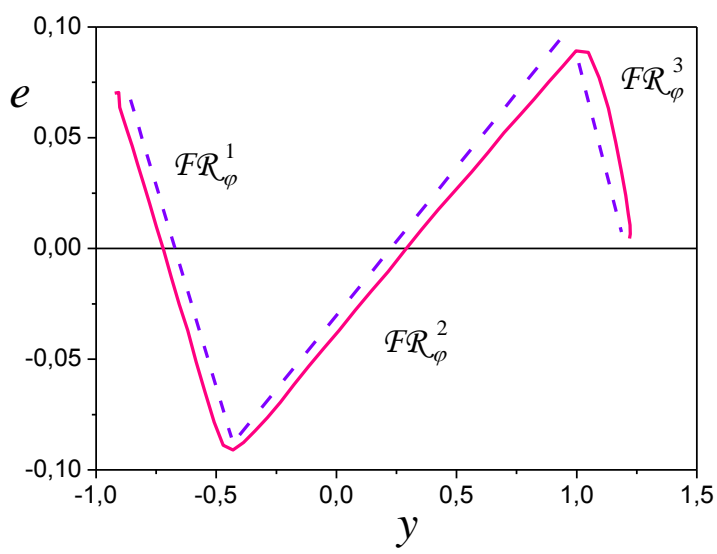

Fig. 5. The upper part $S_{e y}$ on a time gap $[17.2 ; 27.4] \mathrm{s}$

Validity of application an estimation of a change a linear part of function $\varphi(y)$ follows from following statement.

Statement 3. Let: i) in space $\mathscr{P}_{e y}$ the fragment $F R_{p}^{2} \subset S_{e y}$ is described by the equation $e=c y+d$, where $c(\kappa), d$ are some numbers, $\kappa>0$; ii) in space $\mathscr{P}_{k e}$ for $F R_{\varphi, k}^{2} \subset S_{e k}$ the secant $\bar{\gamma}_{2, k}(t)=a_{2, k} k_{s}(t)+b_{2, k}$, where $a_{2, k}, b_{2, k}$ are some numbers, is received; iii) function $\varphi(y)=\operatorname{sat}(y)$ on a fragment $F R_{\varphi}^{2} \subset S_{e y}$ is described by the equation $\varphi(y)=\kappa y$. Then for $c$ on $F R_{\varphi}^{2} \subset S_{e y}$ the estimation $c \leq p / a_{2, k}$ is true, where

$$
p=\left|1-\frac{d}{\delta_{e}}\right|\left|\mu-\frac{b}{\delta_{e}}\right|, \delta_{e}=\max _{t}|e(t)|, \mu=\delta_{e}^{-1} \max _{t} \bar{\gamma}_{2, k}(t) .
$$


Proof. Let $\forall y \in \operatorname{dom}\left(F R_{\varphi}^{2}\right)$

$e=c y+d$.

The fragment $F R_{\varphi, k}^{2} \subset S_{e k}$ in space $\mathscr{P}_{k e}$ corresponds to a fragment $F R_{\varphi}^{2}$. For $F R_{\varphi, k}^{2}$ construct a secant

$$
\bar{\gamma}_{2, k}(t)=a_{2, k} k_{s}(t)+b_{2, k},
$$

where $a_{2, k}, b_{2, k}$ are defined by means of a least square method.

Consider a secant $\bar{\gamma}_{2, k}(t)=a_{2, k} k_{s}(t)+b_{2, k}$. From the secant equation define $y$

$$
y=\frac{a_{2, k} e}{\bar{\gamma}_{2, k}-b_{2, k}} .
$$

Values substitute in (14) and define $c$

$$
\begin{aligned}
c= & \frac{1}{a_{2, k} e}\left(\bar{\gamma}_{2, k}-b_{2, k}\right)(e-d)= \\
& \frac{1}{a_{2, k}}\left(1-\frac{d}{e}\right)\left(\frac{\bar{\gamma}_{2, k}}{e}-\frac{b_{2, k}}{e}\right)
\end{aligned}
$$

Designate $\delta_{e}$ as $\delta_{e}=\max _{t}|e(t)|, \mu=\frac{1}{\delta_{e}} \max _{t} \bar{\gamma}_{2, k}(t)$. Then (15)

$$
c \leq \frac{1}{a_{2, k}}\left(1-\frac{d}{\delta_{e}}\right)\left(\mu-\frac{b_{2, k}}{\delta_{e}}\right) \leq \frac{1}{a_{2, k}}\left|1-\frac{d}{\delta_{e}}\right|\left|\mu-\frac{b_{2, k}}{\delta_{e}}\right| .
$$

As $c=\kappa c_{0}>0$ and $c_{0}>0$, that $a_{2, k}>0$. Last inequality is a positivity $\kappa$ corollary. So, we obtain $c \leq p / a_{2, k}$, where $p$ will satisfy to a condition

$$
p=\left|1-\frac{d}{\delta_{e}}\right|\left|\mu-\frac{b_{2, k}}{\delta_{e}}\right|
$$

Results of structural identification fragments $F R_{\varphi}^{1}, F R_{\varphi}^{3}$ we have obtained above as estimations on parameters $\bar{v}, \underline{v}$ function $\operatorname{sat}(y)$. They will conform to obtained on the basis of the analysis structure $S_{e y}$ qualitative conclusions.

We will describe now algorithm of an estimation a form nonlinear function $\varphi(y)$ on the class $F_{m v}$.

1. Construct structure $S_{e y}$ and divide it into fragments $F R_{\varphi}^{i} \subseteq S_{e y}, i \geq 1$.

2. Estimate on $S_{e y}$ identifiability of system.

3. Construct sector $\operatorname{Sec}\left(F R_{\varphi}^{i}\right)$ for $F R_{\varphi}^{i}$. Make a solution on the class $F_{m v}$. If $\varphi(y) \in F_{m v}$, pass to a step 4 .

4. Make a preliminary solution on a form of function $\varphi(y)$ on the basis of analysis the structure $S_{e y}$.
5. Estimate properties and parameters of function on the basis of the analysis fragments $F R_{\varphi, k}^{i} \subset S_{e k}, i \geq 1$ by means of secant method in space $\mathscr{P}_{k e}$.

6. For $\mathcal{F} R_{\varphi, k}^{i}$ find a secant $\bar{\gamma}_{i, k}$ and a coefficient of determination $r_{e, \bar{\gamma}_{i, k}}^{2}$ corresponding to it.

7. Make a solution on the significance of the obtained parameters of function on values $r_{e, \bar{\gamma}_{i, k}}^{2}$.

Remark 3. The offered approach gives parametric estimations for $\bar{\gamma}_{i, k}$ in the form of some subset belonging to informational set $\mathrm{I}_{o}$. Level of obtained values of estimations is one more measure of adequacy the offered approach.

The final decision about effectiveness of the obtained estimations at a stage of parametric identification system (3) accepts.

The offered approach is based on the analysis of structures $S_{e y}$ and $S_{e k}$. Generally it does not allow identifying any many-valued nonlinearity. Therefore we should fulfil additional researches to consider all features and specificity of nonlinear functions. It confirms an example $S_{e y}$ structure and a phase portrait for a system of the second order. They describe the steady state of a system and are showed on Fig. 6.

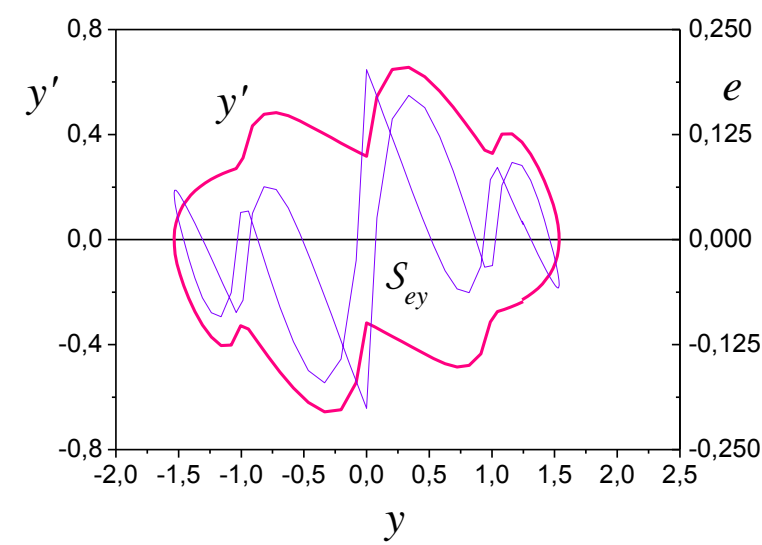

Fig. 6

We see (Fig. 6) that the system is $h$-identified. Nonlinearity has features in points $y=-1, y=0$ and $y=1$. These points are points of change a sign a derivative (see $S_{e y}$-structure). Application of secant method for structures $S_{e k}$ or $S_{e y}$ will show that on intervals of violation of smoothness the coefficient of the slope a secant is close to 0 . The coefficient of determination secants exceeds 0.96 . We conclude that nonlinearity does not contain in a neighbourhood of the specified points of smooth curves. We can suppose that in these points the derivative changes a sign. Explain it as properties of function $\varphi(y)$, and the chosen interval of integration.

Function $\varphi(y)$ has form 


$$
\varphi(y)=\left\{\begin{array}{cc}
2, & \text { if } y>1, \\
\operatorname{sign}(y), & \text { if } y \leq 1 \\
-2, & \text { if } y<-1
\end{array}\right.
$$

The obtained inference confirms Fig. 7. From Fig. 7 we see that the structure $S_{k e}$ contains points of change a sign derivative variable $e$. We consider behaviour of system in space $\mathscr{P}_{k e}$.

Statement 4. Let system (3): i) is $h$-identified; ii) each fragment $F R_{\varphi}^{i}$ of structure $S_{e y}=\bigcup_{i} F R_{\varphi}^{i}, i \geq 1$ contains flexes points. Then the system (3) is not identified in space $\mathscr{P}_{k e}$ if the potency of an applicable domain of each fragment $\mathcal{F R}_{\varphi}^{i}$ does not exceed $n_{i}$, where $n_{i}$ is a positive number.

Proof. We on the basis of the analysis structure $S_{e y}$ obtain set of fragments $F R_{\varphi}^{i}$. Consider a fragment $F R_{\varphi}^{i}$ and a flex point $\mathcal{M}_{i}$ on it. Let $I_{i}(y)$ is a variation interval $y$ between flexes points of fragments $F R_{\varphi}^{i}$ and $F R_{\varphi}^{i+1}$ and $\# I_{i} \leq n_{i}$ where $\# I_{i}$ is a potency of interval $I_{i}$. We suppose that $n_{i}$ is a small number. Therefore it is not enough number of points the data for an estimation of parameters function $\varphi(y)$ by means of secant method. We obtain a low coefficient of determination for a secant of fragment. Statement is proved.

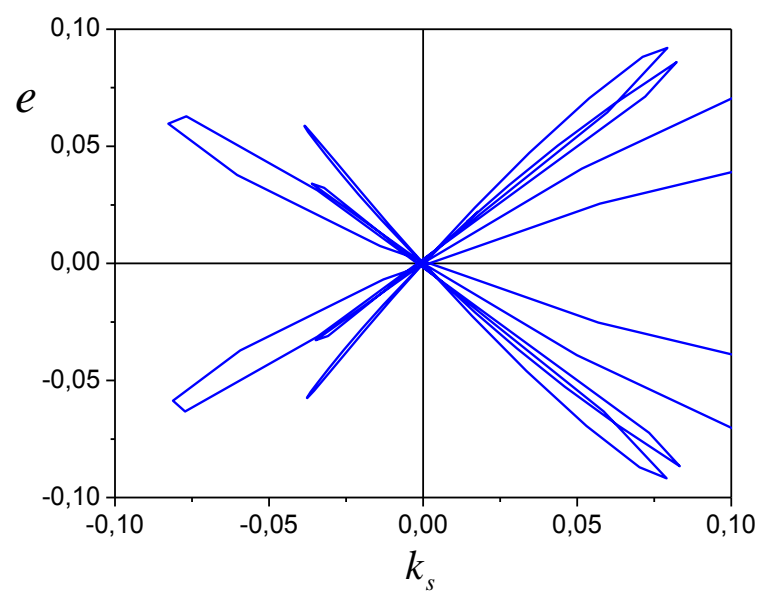

Fig. 7. $S_{k e}$-structure for system with function (16)

\section{C.About Choice of Structure Nonlinearity}

In section 7 we have offered the approach to choice of the class of nonlinear functions. It on construction of sector set and the analysis of properties secants is based. Further, we have considered based on the analysis of properties structure $S_{e y}$ the approach. We have offered algorithms of decision making by means of secant method. Effectiveness of the offered method of the form nonlinearity depends. Properties of structure depend on dynamic properties of system and model of its deriving. The structure $S_{e y}$ is result of approximation motion of system by means of special model. Therefore $S_{e y}$ can contain errors (uncertainty). These errors influence by form a variable $e(t)$ and complicate decision making process. We on an example of system with nonlinearity (16) observed it. Therefore we should offer the approach which will confirm above obtained results in the conditions of a priori uncertainty.

Development of analytical methods decision making in the conditions of a priori uncertainty about structure of system and its properties is a complicated problem. Apply in this case indirect methods. Refer to them a method of frequency bar chart (frequency distribution) which is applied to study of statistical processes. We show, as this method to apply to the analysis of a considered problem.

Consider dynamic system of the second order with nonlinearity (16). On Fig. 6 we showed structures corresponding to it. We name the applied approach the structure-frequency analysis (SFA method). Results of work SFA method in the integrated form are presented on Fig. 8. From Fig. 8 we see that nonlinearity gives non-uniform value distributions $y \in \mathrm{I}_{y}^{i} \subset \operatorname{dom} S_{e y}$. On Fig. 8 we showed one of fragments $S_{e y}$. Designate distribution functions $e, y$ as $f(e), f(y)$.

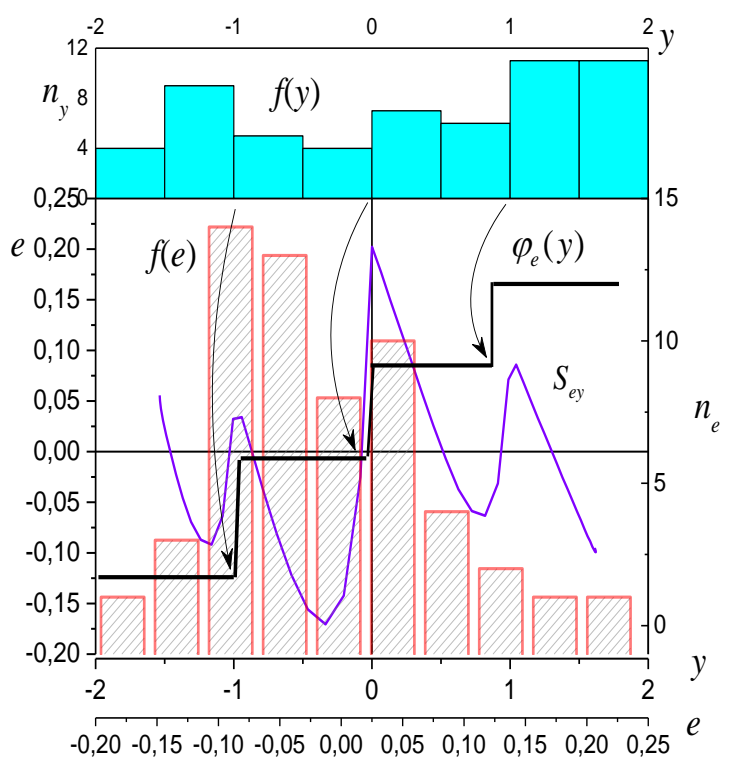

Fig. 8. Results of application of SFA method

Notice that a form of functions $f(y), f(e)$ reflect in corresponding space the form of function (16). The analysis $f(y)$ gives 4 variations intervals of function (16). On Fig. 8 we represent piecewise-linear reconstruction $\varphi_{e}(y)$ of function $\varphi(y)$ in scale to a variable $e$. Arrows we will show choice of switch points of function.

So, we conclude that SFA method allows obtaining an estimation of the form nonlinear function $\varphi(y)$ on the basis of the analysis of functions $n_{e}(e), n_{e}(e)$ and structure $S_{e y}$. The given approach is obvious, but demands from the researcher good knowledge of object domain. This knowledge, eventually, defines final result. 


\section{CONCLUSION}

The method of structural identification dynamic systems with nonlinearity is offered. We showed that for a solution of a problem an estimation structure the condition $h$-identifiability of system should be satisfied. The method of construction the set containing the information on nonlinearity is offered. It is grounded on application of special static model.

The structure $S_{e y}$ is introduced reflecting a change of nonlinear properties of system. The method of estimation the class nonlinear functions on the basis of the analysis sector set for $S_{e y}, S_{e k}$-structures are offered. We showed that in the conditions of a priori uncertainty we can make a conclusion about a form of nonlinear function on $S_{e y}$.

The algorithm of an estimation structure single-valued nonlinear function is offered. We for obtaining of estimations parameters nonlinear function the structure $S_{k e}$ are used. Examples of work algorithm are considered.

The approach to structural identification of manyvalued nonlinearities is described. Existing difficulties are noted. We showed that condition execution $h$ identifiability for some nonlinearity not always is sufficient for an overall estimate of structure nonlinearity. Use the additional information for an improvement of the obtained estimations. It we underline complexity, variety of nonlinearity.

The structurally-frequency method of recovery of nonlinearity is offered. It is the instrument of check solutions and the obtained structural estimations.

The analysis of the obtained results will show the necessity of continuation research for the given direction.

\section{REFERENCES}

[1] V.G. Shashiashvili, "Structural identification of nonlinear dynamic systems on set of the continuous block-oriented models," in XII All-Russia conference on problems of control ARCPC-2014. Moscow on June, 16-19th, 2014. Moscow: V.A. Trapeznikov Institute of Control Sciences, 2014, pp. 3018-3028.

[2] T.H.Van Pelt, and D.S. Bernstein, "Nonlinear system identification using Hammerstein and non-linear feedback models with piecewise linear static maps," International journal control, 2001, vol. 74, no. 18, pp. 1807-1823.

[3] W.J. Rugh, Nonlinear system theory: The Volterra/Wiener approach, The Johns Hopkins University Press, 1981.

[4] G. Dimitriadis, Investigation of nonlinear aeroelastic systems. Thesis of degree the doctor of philosophy. University of Manchester, 2001.

[5] G. Kerschen, K. Worden, A.F. Vakakis, and J.C. Golinval, "Past, present and future of nonlinear system identification in structural dynamics," Mechanical systems and signal processing, 2006, vol. 20, pp. 505-592.

[6] R.Lin, and D.J. Ewins, "Location of localized stiffness non-linearity using measured modal data," Mechanical systems and signal processing, 1995, vol. 9, pp. 329-339.

[7] C.P. Fritzen, "Damage detection based on model updating methods," Mechanical systems and signal processing, 1998, vol. 12, pp. 163-186.
[8] R. Pascual, I. Trendafilova, J.C. Golinval, and W. Heylen, "Damage detection using model updating and identification techniques," in Proceedings of the Second International Conference on Identification in Engineering Systems, Swansea, 1999.

[9] I. Trendafilova, V. Lenaerts, G. Kerschen, J.C. Golinval, J.C., and H. Van Brussel, "Detection, localization and identification of nonlinearities in structural dynamics," in: Proceedings of the International Seminar on Modal Analysis (ISMA), Leuven, 2000.

[10] P. Atkins, and K. Worden, "Identification of a multidegree-of-freedom nonlinear system," in Proceedings of the 15th International Modal Analysis, Conference, Orlando, 1997, pp. 1023-1028.

[11] J.S. Bendat, A.G. Piersol, Random Data: Analysis and Measurement Procedures, third ed. Wiley Interscience: New York, 2000.

[12] G. Kerschen, J.C. Golinval, and F.M. Hemez, "Bayesian model screening for the identification of non-linear mechanical structures," Journal of vibration and acoustics, vol. 125, pp. 389-397, 2003.

[13] Y. Fan, and C.J. Li, "Non-linear system identification using lumped parameter models with embedded feedforward neural networks," Mechanical systems and signal processing, 2002, vol. 16, pp.357-372.

[14] M. Peifer, J. Timmer, and H.U. Voss, "Nonparametric identification of nonlinear oscillating systems," Journal of sound and vibration, 2003, vol. 267, pp. 1157-1167.

[15] H. Yu, J. Peng, and Y. Tang, "Identification of Nonlinear Dynamic Systems Using Hammerstein-Type Neural Network," Mathematical problems in engineering, 2014, vol. 2014, article ID 959507.

[16] A.W. Smith A.W., S.F. Masri, E.B. Kosmatopoulos, A.G. Chassiakos, and T.K. Caughey, "Development of adaptive modeling techniques for non-linear hysteretic systems," International journal of non-linear mechanics, 2002, vol. 37, is. 8 , pp. $1435-1451$.

[17] J. Vörös, "Modeling and identification of hysteresis using special forms of the Coleman-Hodgdon model," Journal of electrical engineering, 2009, vol. 60, no. 2, pp. 100-105.

[18] K. Worden, G. Manson, "On the identification of hysteretic systems, Part I: an extended evolutionary scheme," in Proceedings of the IMAC-XXVIII February 1-4, 2010, Jacksonville, Florida USA. 2010,9p.

[19] M. Peimani, M.J. Yazdanpanah, and N. Khaji, "Parameter estimation in hysteretic systems based on adaptive leastsquares," Journal of information systems and telecommunication, 2013, vol. 1, no. 4, pp. 217-221.

[20] Y. Tan, R. Dong, H. Chen, and H. He, "Neural network based identification of hysteresis in human meridian systems," Int. J. Appl. Math. Comput. Sci., 2012, vol. 22, no. 3, pp. 685-694.

[21] Y. Ding, B.Y. Zhao, and B. Wu, "Structural system identification with extended Kalman filter and orthogonal decomposition of excitation," mathematical problems in engineering, 2014, vol., article ID 987694, 10 p.

[22] N.N. Karabutov, "Selection of the structure of a model in processing the results of measurements in control systems," Measurement techniques, 2008,vol. 51, no. 9, pp. 960-966.

[23] N.N. Karabutov, Structural Identification of Systems. Analysis of Information Structures. Moscow: URSS/ Librokom, 2009.

[24] N.N. Karabutov, Structural identification of static objects: Fields, structures, methods. Moscow: Librokom, 2011.

[25] N.N. Karabutov, "Structural identification of nonlinear static system on basis of analysis sector sets," International 
journal of intelligent systems and applications, 2014, vol. 6, no. 1. pp. 1-10.

[26] V.S. Pugachev, Automatic control fundamentals. Moscow: Nauka, 1968.

[27] Y.E. Kazakov, B.G. Dostupov, Statistical dynamics of nonlinear automatic systems. Moscow: Fizmatgis, 1962.

[28] V.D. Furasov, Stability of motion, estimation and stabilization. Moscow: Nauka, 1977.

[29] Choquet G. L'enseignement de la geometrie. Paris: Hermann, 1964.

[30] N.N. Karabutov, Method of structural identification nonlinear plants. Saarbrucken: Palmarium Academic Publishing, 2014.

[31] F. Mosteller, and J. Tukey, Data analysis and regression: A second course in statistics. Addison-Wesley Publishing Company. 1977.

[32] M. Young, The Technical Writer's Handbook. Mill Valley, CA: University Science, 1989.

\section{Author's Profiles}

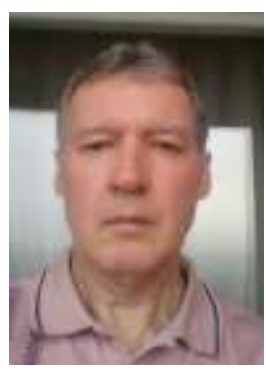

Nikolay Karabutov is the professor of department Problem Control of Moscow state engineering university of radio engineering, Electronics and automation. The professor department of mathematics Financial University under the Government of the Russian Federation. Dr.Sci.Tech. The research areas are the automatic control theory, identification, adaptive control and decision-making.

How to cite this paper: Nikolay Karabutov,"Structural Identification of Nonlinear Dynamic Systems", International Journal of Intelligent Systems and Applications (IJISA), vol.7, no.9, pp.1-11, 2015. DOI: 10.5815/ijisa.2015.09.01 